\title{
Driver Fatigue Detection System Based on DM3730
}

\author{
Ming Cai ${ }^{1,2, *}, \mathrm{Ye} \mathrm{Gu}^{3}$, Haixin $\mathrm{Sun}^{3}, \mathrm{Jie}^{\mathrm{Q}}{ }^{3}$ and Boliang Wang ${ }^{1}$ \\ ${ }^{1}$ School of Information Science and Engineering, Xiamen University, China \\ ${ }^{2}$ School of Information Science and Technology, Xiamen University Tan KahKee College, China \\ ${ }^{3}$ Key Laboratory of Underwater Acoustic Communication and Marine Information Technology (Xiamen University), \\ Ministry of Education, Xiamen, China
}

\begin{abstract}
Driver fatigue is a popular problem which has attracted people's views. Many research departments are researching driver fatigue detection in order to improve the traffic safety. This paper presented a driver fatigue detection system based on DM3730. The system calculated the inter-image difference between frames captured by near-infrared light irradiation, which included identification of the eyes by Otsu adaptive threshold segmentation method and prediction of the orientation of the eye in nearby images by Kalman filter. Then the system determined the state of fatigue by improved PERCLOS (Percentage of Eyelid Closure over the Pupil)method. Experimental results show that the system has the advantages of small size and low power consumption. Meanwhile it meets the requirements of all-weather, real-time monitoring. The system can be extended to automobile and other production processes which the fatigue monitoring is required.
\end{abstract}

Keywords: Driver fatigue detection, Kalman filter, PERCLOS.

\section{INTRODUCTION}

The methods of detecting driver fatigue include physiological signal detection $[1,2]$, vehicle behavioral characteristics detection [3] and computer vision based detection [4-6]. In these driving fatigue evaluation methods, PERCLOS (Percentage of Eyelid Closure Over the Pupil Over Time) based on computer vision is recognized as the most effective, automotive and real-time one [7], which was first proposed in the technical forum of the Federal Highway Administration in April 1999. The PERCLOS method regards the time percentage of closing eyes as a key indicator of driver fatigue predictions.

Although there are a variety of methods of real-time driver fatigue detection, most of them are limited to theoretical research. Some devices have a lot of limitations, while others have many problems to be solved. The current researches of vehicle driver fatigue detection monitoring focus on improving the characteristic of real-time, robustness, accuracy, cost performance and multi-feature fusion.

This paper designed an all-weather driver fatigue detection system to determine the fatigue level of drivers by extracting the pupil characteristics. This system built on TI's DaVinci 3730 platform is visual, real-time and noncontacting.

\footnotetext{
*Address correspondence to this author at the School of information science and engineering, Xiamen University, China; Tel: 008615959250539 ; E-mail: hxsun@xmu.edu.cn
}

\section{SYSTEM OVERVIEW}

\subsection{Hardware Structure}

This system based on DM3730 hardware platform collects the reflection lights of the face of the driver by $850 \mathrm{~nm}$ infrared cameras with OV7725 sensors. Then the system analyzes the eye condition by the difference of inter-frame images, and determines the extent of the driver's fatigue by the red-eye effect of the eye in the infrared radiation generated by the light source. System hardware structure is shown in (Fig. 1).

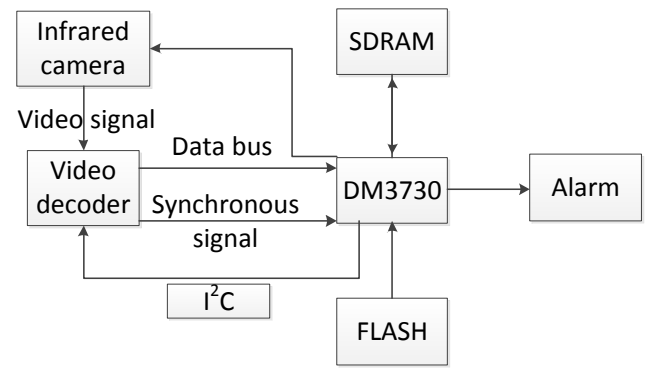

Fig. (1). Hardware structure.

\subsection{Software Structure}

Software structure of the system is composed by image processing algorithms, including image difference method to analyze the different brightness of the pupil in adjacent frames, estimating the pupil's position in the image by the previous one, extracting the feature parameters of the eye to count the value of discrete PERCLOS. The series of algo- 
rithms can determine whether the driver is fatigue driving. System software structure is shown in Fig. 2.

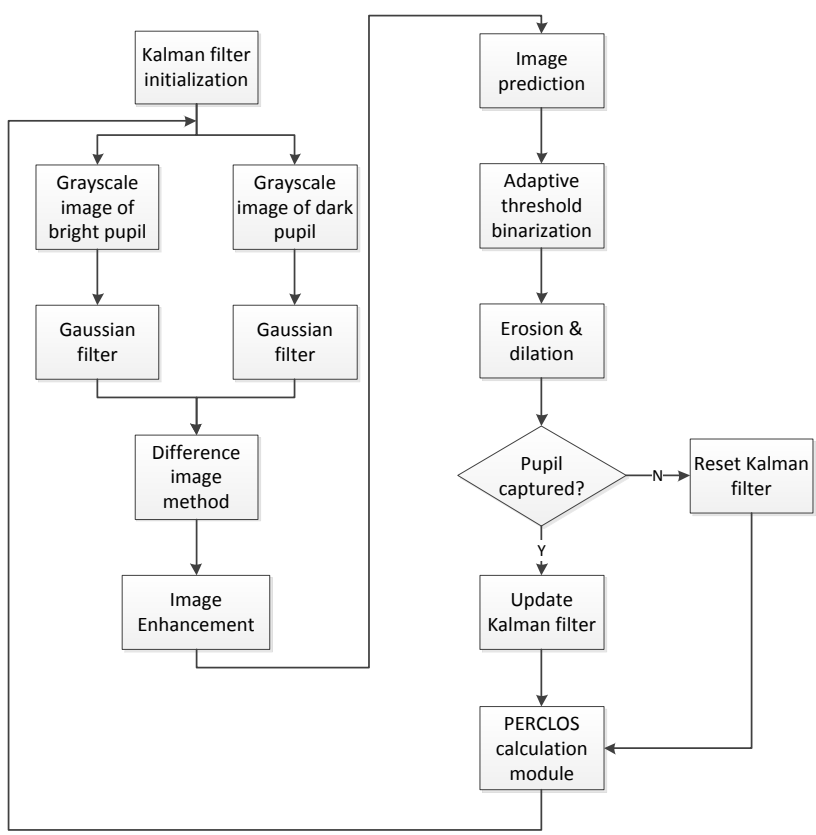

Fig. (2). Software structure

\section{FATIGUE DETECTION ALGORITHM}

\subsection{Image Acquisition}

Image acquisition module gets real-time images of driver's face from LED light source. As shown in Fig. 3, the device set includes near-infrared LED light sources, a light source control device, a device accommodating box, an OV7725 sensor camera, and camera lens with the infrared band-pass filters.

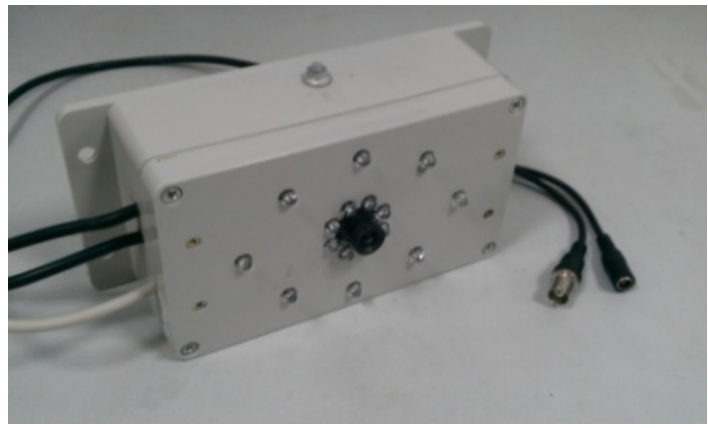

Fig. (3). Image acquisition device.

The light source is inner and outer rings of near-infrared wavelength of $850 \mathrm{~nm}$ LED light sources on a circuit board. Eight LED lights in the inner ring around the camera lens produce red-eye effect of the driver's eyes, while eight lights in the outer ring are relatively dispersed to avoid red-eye effect. The brightness of the inner and outer lights is same by adjusting the rheostat in the lights. The system uses OV7725 sensor camera that can keep a good performance of image acquisition in low illumination and can get a small motion deviation between frames.

\subsection{Eyes Localization}

Image acquisition system determines the position of the driver's eyes from two nearby frames. The brightness of the eyes in the images is different. The eyes location can be acquired from a series of processing including image difference and image screening methods.

LED lights in the inner ring flash to acquire images of bright pupil, which is called red-eye images. LED lights in the outer ring generate normal images of dark pupil. The calculation formula to obtain the difference image is shown in (1).

$C(i, j)=|L(i, j)-A(i, j)|$

$\mathrm{L}(\mathrm{i}, \mathrm{j})$ is the dark pupil image. $\mathrm{A}(\mathrm{i}, \mathrm{j})$ is the bright pupil image. $C(i, j)$ is the difference image. The difference image is the absolute value of the difference of all the pixels in the dark and bright images. In (Fig. 4), (c) is the difference results of (a) and (b). It can be observed from the difference image that the luminance of the pupil region is so lighter than other regions that the difference is significant between the images.

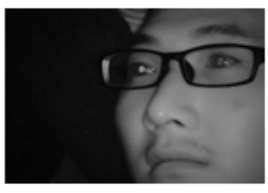

(a) bright pupil image

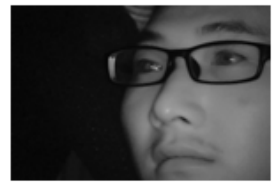

(b) Dark pupil image

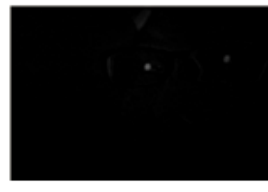

(c) Difference image
Fig. (4). Image difference operations.

In order to reduce image interference in subsequent processing, the system uses OTSU method [8]. According to the characteristics of the grayscale image, the adaptive thresholding method divides the image into two parts: background and foreground. The class variance between the foreground and background reaches the maximum after image binarization by threshold. The difference image to be processed contains 256 gray levels $(0,1, \ldots, 255)$. Let $i$ be the gray value of the pixel, the number of pixels be $\mathrm{Ni}$, and the ratio in the total pixels be $\mathrm{Pi}$. The whole image is divided into dark area $\mathrm{C} 1$ and bright area $\mathrm{C} 2$ by threshold t. The variance $\sigma$ is a function of $\mathrm{t}$.

$\sigma=c_{1} \times c_{2} \times\left(u_{1}-u_{2}\right)^{2}$

where $c_{1}=\sum_{\mathrm{i}=0}^{\mathrm{t}} P_{i}, c_{2}=1-\mathrm{c} 1, u_{1}=\sum_{\mathrm{i}=0}^{\mathrm{t}}\left(\mathrm{i} \times P_{i}\right) / c_{1}, u_{2}=$ $\sum_{\mathrm{i}=\mathrm{t}+1}^{\mathrm{L}-1}\left(\mathrm{i} \times P_{i}\right) / c_{2}$. Select the best threshold value $\mathrm{T}$ to obtain the maximum value of $\sigma$ in (2) when $t=T$.

The segmentation results based on the OTSU adaptive threshold method are shown in (Table 1).

It can be verified from the segmentation results that the system greatly enhances the ability to adapt to the environment by introducing an adaptive segmentation threshold. In order to eliminate image noise after adaptive thresholding segmentation, an open operation is implemented, which corrodes first then expands. The corrosion operation uses a $2 \times 2$ template (3).

$\left[\begin{array}{ll}1 & 1 \\ 1 & 0\end{array}\right]$ 
Table 1. Segmentation results based on OTSU method.

\begin{tabular}{|l|l|c|c|}
\hline Original Image & Difference Image & Threshold & Binarization \\
\hline \hline & & 13 & $\cdot$ \\
\hline & & & \\
\hline & & 58 & \\
\hline & & & \\
\hline & & & \\
\hline & & & \\
\hline
\end{tabular}

The binary image is corroded by this template. The result is shown in Fig. 5b. The expansion operation uses a $3 \times 3$ template (4).

$$
\left[\begin{array}{lll}
1 & 1 & 1 \\
1 & 1 & 1 \\
1 & 1 & 1
\end{array}\right]
$$

The image after expansion operation is shown in Fig. 5c.

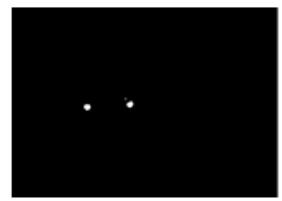

(a) The pupil region

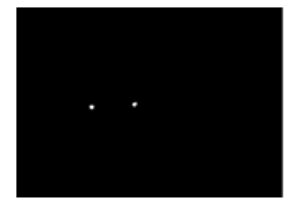

(b) Image after corrosion

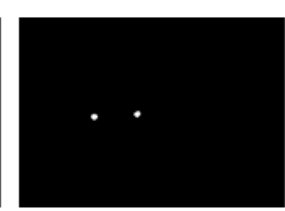

(c) Image after expansion
Fig. (5). Open operation of binary image.

After the open operation, the pupil regions are kept in the image. The two largest connected regions in the image can be regarded as the pupil regions within the limited scope by the Kalman filter.

The system uses a Kalman filter to predict the eye position in the next frame by the detection result of the current frame, which reduces the size of the image processing and improves accuracy. The operation process of Kalman filter is shown in Fig. 6.

In the pupil tracking process, let the motion parameters of system state $x_{k}$ be $x, y, d x, d y$, according to the eye status, where $\mathrm{x}$ is the horizontal ordinate of the center of the pupil, $\mathrm{y}$ is the vertical axis, $\mathrm{dx}$ is the velocity in $\mathrm{x}$-axis, and dy is the velocity in $\mathrm{y}$-axis. Define $(\mathrm{x}, \mathrm{y})^{\mathrm{T}}$ as the observation vector. The system parameters are defined as follows based on the assumption state.

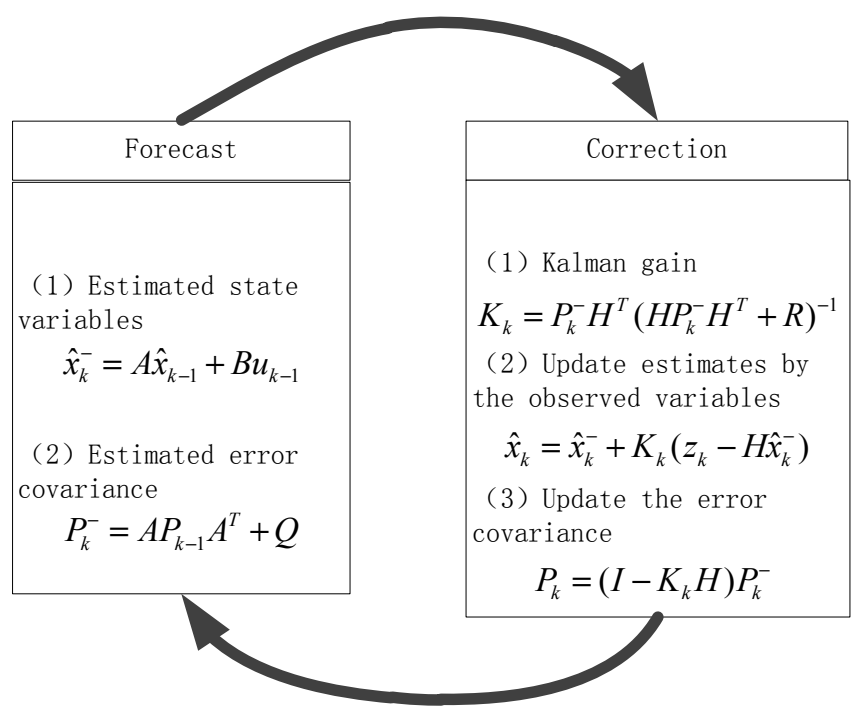

Fig. (6). Process of Kalman filter.

Transition matrix of system status (5):

$A=\left[\begin{array}{cccc}1 & 0 & d t & 0 \\ 0 & 1 & 0 & d t \\ 0 & 0 & 1 & 0 \\ 0 & 0 & 0 & 1\end{array}\right]$

Observation matrix (6):

$H=\left[\begin{array}{llll}1 & 0 & 0 & 0 \\ 0 & 1 & 0 & 0\end{array}\right]$

Noise covariance matrix of system status (7):

$Q=\left[\begin{array}{cccc}0.01 & 0 & 0 & 0 \\ 0 & 0.01 & 0 & 0 \\ 0 & 0 & 0.01 & 0 \\ 0 & 0 & 0 & 0.01\end{array}\right]$

Noise covariance matrix of observation (8):

$R=\left[\begin{array}{ll}0.2845 & 0.0045 \\ 0.0045 & 0.0455\end{array}\right]$

Error covariance matrix (9):

$P=\left[\begin{array}{cccc}100 & 0 & 0 & 0 \\ 0 & 100 & 0 & 0 \\ 0 & 0 & 100 & 0 \\ 0 & 0 & 0 & 100\end{array}\right]$

The pupil tracking steps by Kalman filter are as follows:

1. Initialize the system state.

2. Try to extract the pupil in the k-th frame in accordance with the pupil region in the (k-1)-th frame. If successful, then modify the system state parameters of Kalman filter. If failed, then set the search region to the whole image and restart Kalman filtering process in the next frame.

3. Predict the motion state of the next frame by Kalman filter. Narrow the extraction range of the eigenvalues. 


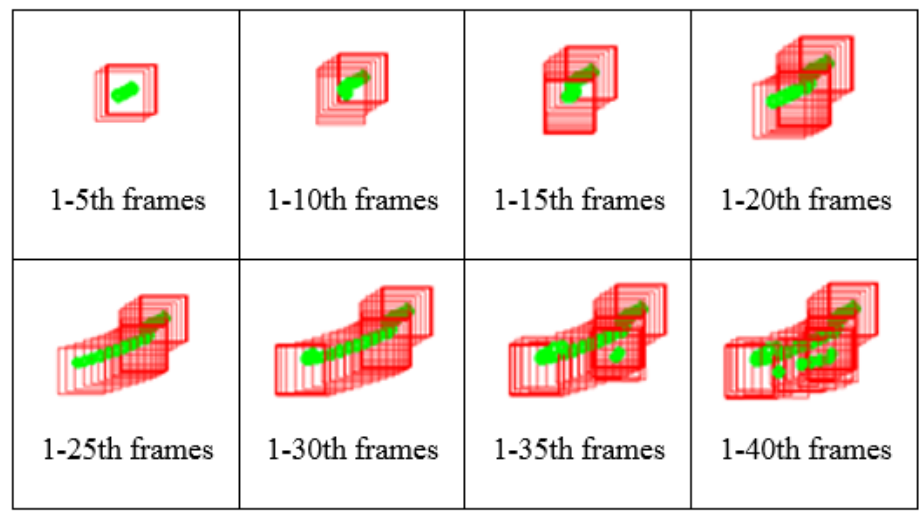

Fig. (7). Pupil tracking results.

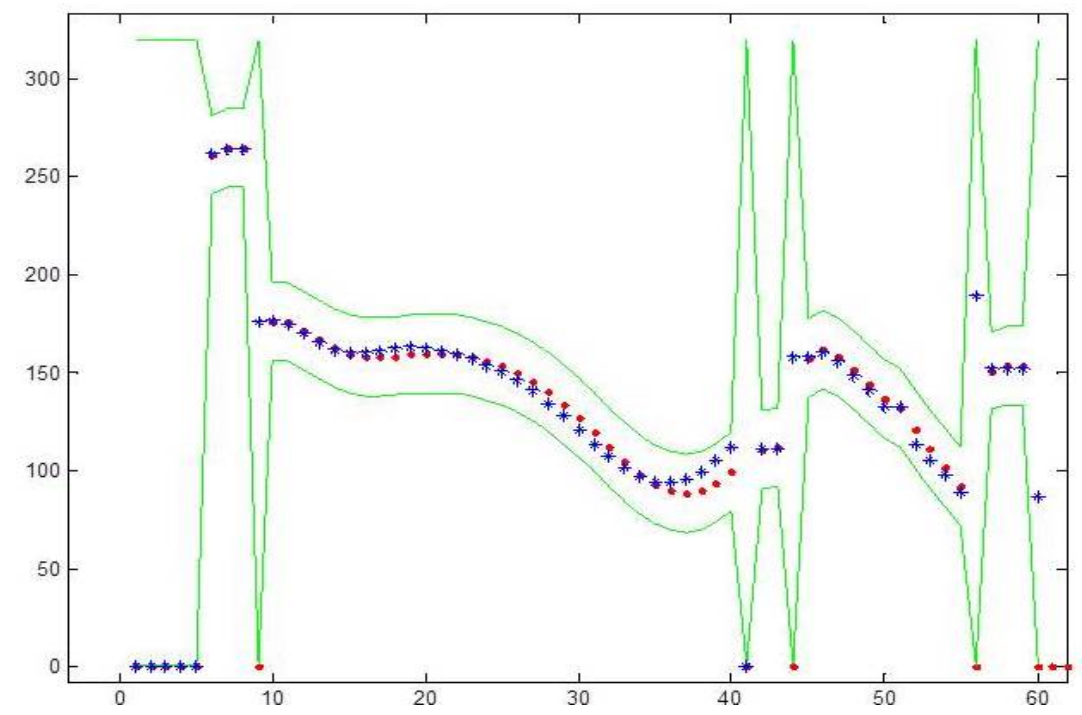

Fig. (8). Pupil trajectory of Kalman filter.

\section{Return to step 2.}

The pupil tracking results are shown in Fig. 7.

In Fig. 7., the green spots are the positioning trajectories of the pupil, while the red frames are the region sets that can predict the position of the pupil in the next frame. As can be seen from $1-30^{\text {th }}$ frames, the Kalman filter works well that accurately predicts which region the pupil will appear in the next frame. In $30-35^{\text {th }}$ frames, the pupil position has a large deviation. The probable reason is the fast-moving of the driver's eyes or frame loss. When the system fails to find the pupil in the predicting frame, the Kalman filter will be reset, and the search region is also reset to the entire frame. The recapture and predict situations are shown in $30-40^{\text {th }}$ frames.

As the trajectory of the pupil can be seen in Fig. 8, the Kalman filter can predict the possible region in the next frame, in which the pupil can be found in most cases. When the pupil position comes out of the predicting frame (as described in the $9^{\text {th }}$ and $44^{\text {th }}$ points), the Kalman filter is reset.

As can be seen in Fig. 9, the Kalman filter can predict the correct position of the pupil in the next frame, which not only reduces the interference caused by other regions but also reduces the calculation of feature extraction. The timeliness and robustness of the system are greatly improved.

\subsection{Eye Feature Extraction}

After extracting the contour of the eye region, the characteristic parameters (for example, the aspect ratio and the area of the eyes) can be solved. By analyzing these parameters, it is a relatively simple, feasible and effective way to identify the eye state. Calculate the area and the aspect ratio of the circumscribed rectangle of the region. The driver's eyes are determined to be closed when the area is smaller than the threshold value.

\subsection{Fatigue Analysis and Monitoring}

Among all the non-contact driver fatigue monitor methods, PERCLOS criterion is one of the most effective internationally recognized fatigue detection methods. There are three evaluation indicators in PERCLOS method: P70, P80, and EM [9-11]. We apply P80 as the criteria of driver fatigue detecting, which believes the driver's eyes closed when more than $80 \%$ area of the pupil is covered by the eyelids. Measurement principle of discrete PERCLOS value is shown in Fig. 10. 

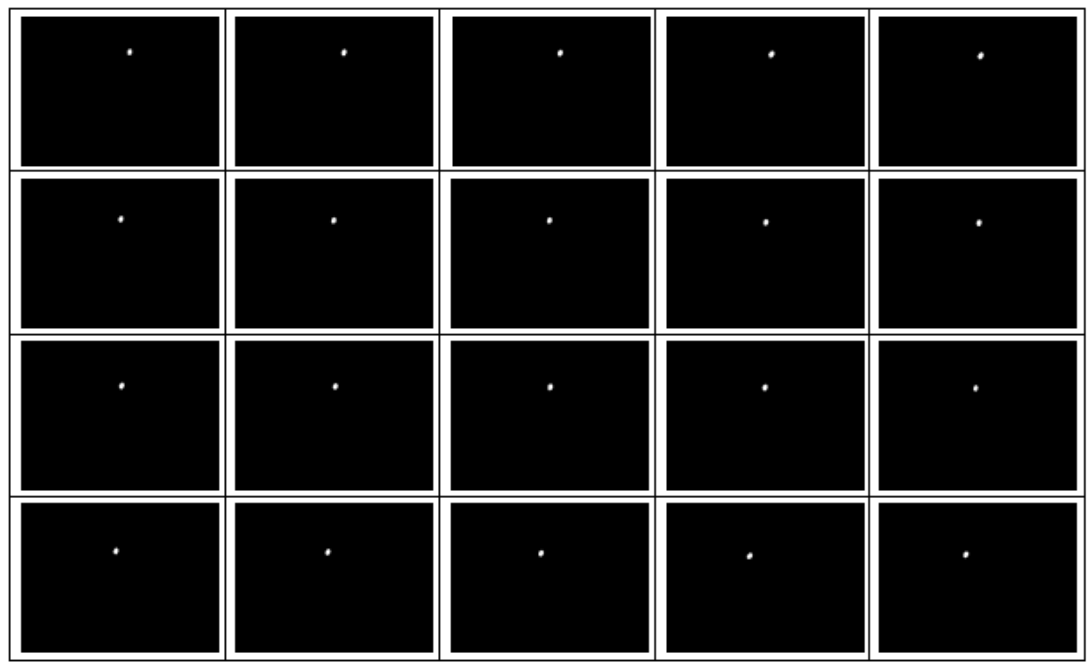

Fig. (9). Pupil inspections of consecutive frames.

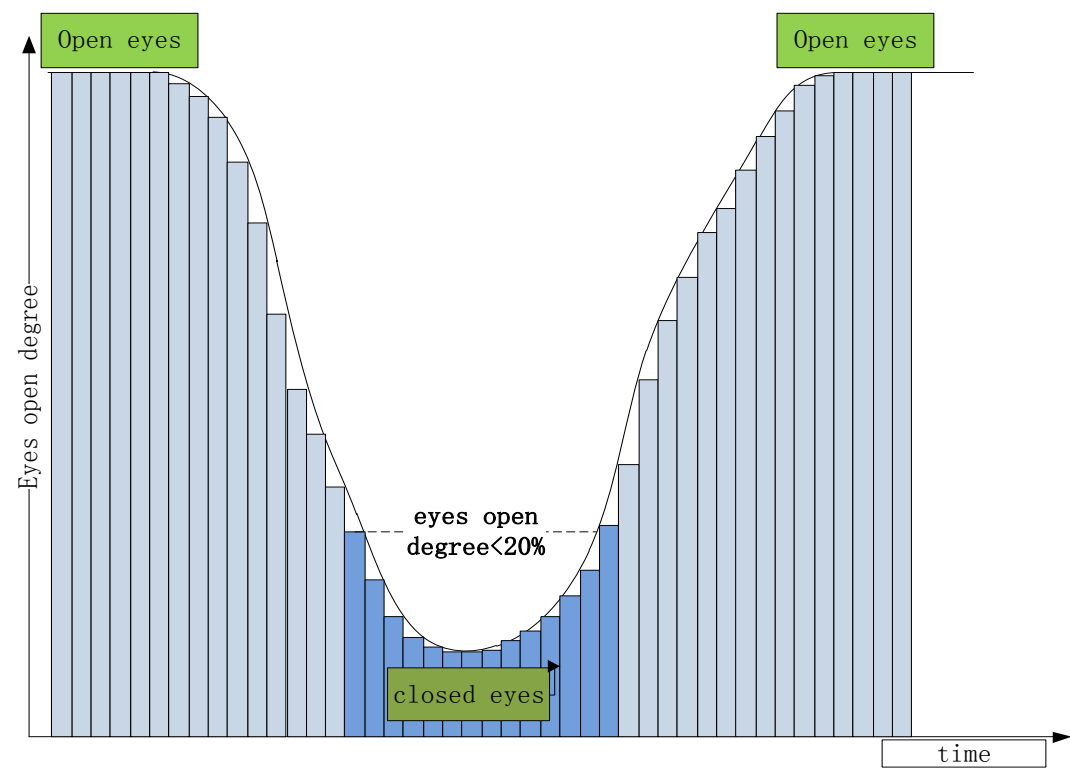

Fig. (10). Measurement principle of discrete PERCLOS value.

The statistical method of discrete PERCLOS value is shown in (10).

PERCLOS $=\frac{\sum_{t=1}^{n} t_{i}}{T}$

$\mathrm{T}$ is the sampling duration, $\mathrm{n}$ is the count of eyes closing in the sampling duration, and $t_{i}$ is time duration of the $i$-th eyes closed. By (10), the PERCLOS value can be calculated that represents the degree of fatigue of the driver.

\section{EXPERIMENTAL RESULTS}

Image capture frequency in acquisition system is $18 \mathrm{fps}$. The image size is $320 \times 240$. The test driver simulates two states of normal driving and fatigue driving, where in the state of normal driving the driver's head maintains straight ahead and occasionally turned around to see the rearview mirror.
Experimental results show that the accuracy of the system to detect driver fatigue reaches more than $82 \%$, of which the accuracy to position human eyes reaches more than $92 \%$. Besides, the accuracy of the system to position human eyes is higher in dark environments than in bright environments, while the calculation gets reduced.

\section{CONCLUSION}

In this paper, we present a driver fatigue detection system based on DM3730. The system accesses the images of the driver's face by the image acquisition device. The nearinfrared light produces the red-eye effect. The difference image is generated from adjacent frames. Then the pupil is segmented based on OTSU adaptive threshold segmentation method. The pupil position is predicted from the neighboring 
frame by a Kalman filter. In the final step, the discrete PERCLOS value is calculated to detect the driver fatigue condition.

The fatigue detection system works not only in the daytime but also at night. The unified feature extraction method is accurate and insensitive to the environment. Compared to the feature extraction method of pattern recognition, the algorithm designed for the using in the environment of this system is simple, efficient and accurate, improving the shortcomings of pattern recognition and laying the foundation for large-scale promotion.

\section{CONFLICT OF INTEREST}

The authors confirm that this article content has no conflict of interest.

\section{ACKNOWLEDGEMENTS}

This work is supported by the natural science foundation of Fujian Province (No. 2013J01258), the key projects of Fujian Province (No. 2012H1012), the national natural science foundation of China (No. 61107023) and the Ph. D. programs foundation of ministry of education of China (No. 20110121120020).

\section{REFERENCES}

[1] M. Simon, E. A. Schmidt, W. E. Kincses, M. Fritzsche, A. Bruns, C. Aufmuth, M. Bogdan, W. Rosenstiel, and M. Schrauf, "EEG alpha spindle measures as indicators of driver fatigue under real traf- fic conditions,"Clinical Neurophysiology, vol. 122, pp. 1168-1178, 2011.

[2] M. Patel, S. K. L. Lal, D. Kavanagh, and P.Rossiter, "Applying neural network analysis on heart rate variability data to assess driver fatigue," Expert Systems with Applications, vol. 38, pp. 72357242, 2011.

[3] E. Dagan, O. Mano, G. P. Stein, and A. Shashua, "Forward collision warning with a single camera," In: Intelligent Vehicles Symposium, 2004, pp. 37-42.

[4] A. Bhardwaj, P. Aggarwal, R. Kumar, and N. Chandra, "Image Filtering Techniques Used for Monitoring Driver Fatigue: A Survey," International Journal of Scientific and Research Publications, vol. 3, 2013, pp. 702-705.

[5] X. Q. Luo, R. Hu, and T. E. Fan, "The driver fatigue monitoring system based on face recognition technology,"Intelligent Control and Information Processing (ICICIP), $20134^{\text {th }}$ International Conference, Beijing, China, 2013, pp. 384-388.

[6] C. H. Zhao, J. Lian, Q. Dang, and C. Tong, "Classification of driver fatigue expressions by combined curvelet features and Gabor features, and random subspace ensembles of support vector machines," Journal of Intelligent and Fuzzy Systems, vol. 26, pp. 91100, 2014.

[7] M. H. Sigari, "Driver hypo-vigilance detection based on eyelid behavior," In: $7^{\text {th }}$ International Conference on Advances in Pattern Recognition (ICAPR 2009), 2009, pp. 426-429.

[8] T. Pradhan, A.N.Bagaria, and A. Routray, "Measurement of PERCLOS using eigen-eyes," In: $4^{\text {th }}$ International Conference on Intelligent Human Computer Interaction (IHCI), 2012, pp. 1-4.

[9] B. Alshaqaqi, A. S. Baquhaizel, M. El A. Ouis, M. Boumehed, A. Ouamri, and M. Keche, "Driver drowsiness detection system", In: $8^{\text {th }}$ International Workshop on Systems, Signal Processing and their Applications (WoSSPA), 2013, pp. 151-155.

[10] N. Ostu, "A threshold selection method from gray-level histogram," IEEE Trans, vol. 9, pp. 62-69, 1979.

[11] J. L. Wan,"Research on bias control and optimum operating condition of avalanche photodetector,'In: International Conference on Electronic Measurement \& Instruments (ICEMI), $20036^{\text {th }}$ International Conference, Taiyuan, China, 2003, pp. 1684-1687.

(C) Cai et al.; Licensee Bentham Open.

This is an open access article licensed under the terms of the (https://creativecommons.org/licenses/by/4.0/legalcode), which permits unrestricted, non-commercial use, distribution and reproduction in any medium, provided the work is properly cited. 